\title{
Article \\ Study on the Rotor Strength of High-Speed Permanent Magnet Motor Considering the Influence of Assembly Pressing Force
}

\author{
Yusheng $\mathrm{Hu}^{1,2,3}$, Liyi $\mathrm{Li}^{1, *}$, Weilin Guo ${ }^{2,3}$ and Shanshan Wang ${ }^{2,3} \mathbb{C}$ \\ 1 School of Electrical Engineering and Automation, Harbin Institute of Technology, Harbin 150006, China; \\ cm_institute_0@cn.gree.com \\ 2 Gree Electric Appliances, Inc. of Zhuhai, Zhuhai 519000, China; gwl_gree@126.com (W.G.); \\ msi_institute@cn.gree.com (S.W.) \\ 3 Guangdong Provincial Key Laboratory of High-Speed and Energy Conservation Motor System, \\ Zhuhai 519000, China \\ * Correspondence: liliyi@hit.edu.cn; Tel.: +86-0756-8589876
}

Citation: $\mathrm{Hu}, \mathrm{Y} . ; \mathrm{Li}, \mathrm{L} . ; \mathrm{Guo}, \mathrm{W}$; Wang, S. Study on the Rotor Strength of High-Speed Permanent Magnet Motor Considering the Influence of Assembly Pressing Force. Symmetry 2021, 13, 2161. https://doi.org/ $10.3390 /$ sym 13112161

Academic Editor: Jan Awrejcewicz

Received: 12 October 2021

Accepted: 2 November 2021

Published: 11 November 2021

Publisher's Note: MDPI stays neutral with regard to jurisdictional claims in published maps and institutional affiliations.

Copyright: (C) 2021 by the authors Licensee MDPI, Basel, Switzerland. This article is an open access article distributed under the terms and conditions of the Creative Commons Attribution (CC BY) license (https:// creativecommons.org/licenses/by/ $4.0 /)$.

\begin{abstract}
In engineering application, the hot press assembly technology is often used to improve the stability of the rotor structure, but the conventional design methods cannot effectively evaluate the influence of this process on the rotor strength, which easily causes the rotor strength to exceed its safety margin range, and seriously it will lead to the failure of the rotor structure. This paper takes the cylindrical magnet surface-mounted high-speed permanent magnet synchronous motor rotor as the research object. Firstly, the influence of the assembly pressing force on the rotor stresses and interference is analyzed; then, comprehensively considering the assembly pressing force, speed and temperature, the rotor strength's design method with high structural stability is proposed. Finally, based on the proposed method, the rotor strength of a $100 \mathrm{~kW} / 30,000 \mathrm{rpm}$ high-speed motor is designed, and the feasibility of the design is verified by over-speed experiment.
\end{abstract}

Keywords: hot press assembly technology; assembly pressing force; rotor strength; over-speed experiment

\section{Introduction}

Permanent magnet synchronous motor has the advantages of small volume, high efficiency, and high power density, and is widely used high-speed motor, such as centrifugal compressors, air compressors, blowers and flywheel energy storage systems. However, the tensile strength of permanent magnet materials is low and easily damaged by highspeed centrifugal force, so the rotor strength design of high-speed permanent magnet synchronous motor is particularly important. According to the location of permanent magnet on the rotor, the permanent magnet synchronous motor rotor mainly has two different structures: surface-mounted and buried. Due to the strength limitation of silicon steel sheets, the application of buried rotor in the high-speed field is relatively smaller than surface-mounted rotor. In order to ensure the surface-mounted permanent magnet rotor strength at high speed, the sleeve and permanent magnet usually adopt an interference fit. If the interference is too small, the sleeve will loosen at high speed, and the permanent magnet is easily damaged by centrifugal force; if the interference is too large, the sleeve stress will easily exceed its maximum allowable stress, and there is a problem of assembly difficultly. Therefore, the reasonable interference is very important to ensure the rotor strength of high-speed motor.

Nowadays, the research on high-speed permanent magnet motor rotor's interference and strength has been relatively comprehensive. The analytical solution of the segmented permanent magnet rotor strength was proposed in [1], and the impacts of speed, interference and sleeve thickness on rotor strength were investigated. The analytical solution of the solid cylinder permanent magnet rotor's stresses, displacement and interference were 
proposed in [2], and compared with the results of finite element method, the analytical formula proposed can accurately predict rotor's stresses and displacement when high speed and temperature rising were considered simultaneously.

The influences of speed, temperature rising on interference between permanent magnet and sleeve were analyzed in [3] by theoretical analysis and finite element method respectively, and the author researched the impacts of speed on the contact pressure between permanent magnet and sleeve by finite element method. The $400 \mathrm{~kW} / 10,000 \mathrm{rpm}$ high-speed permanent magnet generator for the waste heat power system's turbine was analyzed in [4] by the multiphasic coupling, and the variation of rotor stresses along with interference and carbon fiber sleeve thickness was studied.

The influences of sleeve thickness, interference on rotor stresses under high-speed cold state and hot state were analyzed in [5], and the author designed sleeve thickness by comprehensively considering the rotor's mechanical stresses, critical speed and unbalance vibration response. The carbon fiber rotor stresses considering speed, temperature and interference were resolved in [6] by the 2D isotropic model, and so as to ensure the magnet and fiber's structural integrity, glass fiber was used between magnet and carbon fiber sleeve and at the two ends of magnet to avoid carbon fiber sleeve's bend. Comprehensively considering the electromagnetic, thermal and mechanical characteristics, The $100 \mathrm{~W} / 500,000 \mathrm{rpm}$ permanent magnet generator for gas turbines was designed in [7], and during mechanical analysis, the steel and titanium rotor stresses were compared at $23{ }^{\circ} \mathrm{C}$ and the interference of $15 \mu \mathrm{m}$ for titanium but $10 \mu \mathrm{m}$ for steel. The interference and mechanical stresses of enclosure as well as permanent magnet were analyzed in [8], and based on thick walled theory, the author presented the analytical method of mechanical stresses and interference, which was in good agreement with finite element method.

Considering the mutual constraints of mechanical strength, rotor dynamics, mechanical losses and thermal field, the $100 \mathrm{~kW} / 32,000 \mathrm{rpm}$ high-speed permanent magnet motor was designed in [9], and the author analyzed the rotor stresses' variation along with interference and the relation between sleeve thickness and interference. The design method of coupling electromagnetic and mechanical is proposed in [10] for designing the $200 \mathrm{~kW} / 40,000 \mathrm{rpm}$ high-speed permanent magnet motor, and the author designed the minimum sleeve thickness of Inconel and Ti6Al4V by comprehensively considering interference and the thickness of sleeve and permanent magnet.

Most studies do not consider the influence of the assembly technology on rotor strength in engineering application. This paper will take the cylindrical magnet surfacemounted high-speed permanent magnet synchronous motor rotor (Figure 1) as the research object to study the rotor strength's design method considering the influence of assembly pressing force caused by the hot press assembly technology (Figure 2), which includes the impact of assembly pressing force on the interference and the rotor stresses, while taking into account the influences of speed, temperature rise and interference on the rotor strength.

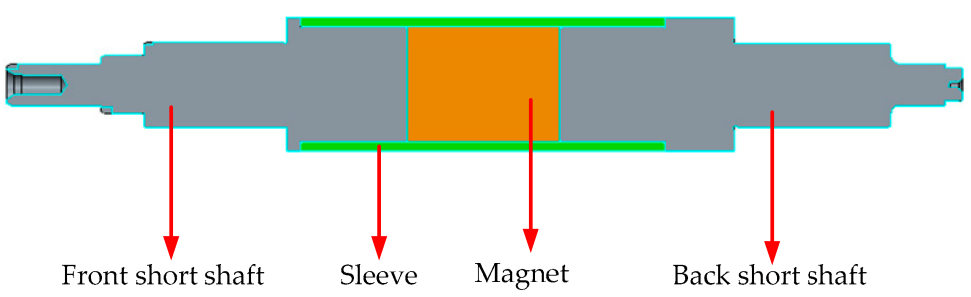

Figure 1. Schematic diagram of cylindrical magnet surface-mounted permanent magnet motor's rotor structure. 


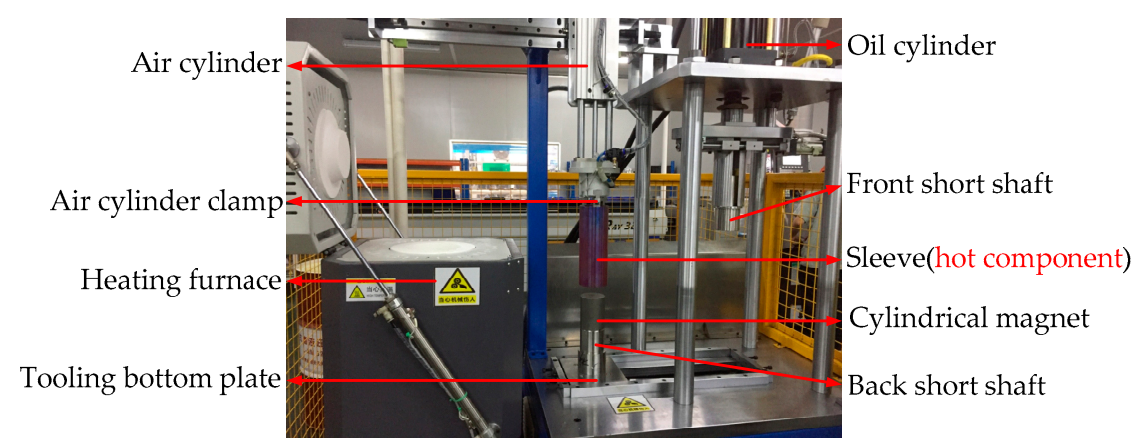

Figure 2. The cylindrical magnet surface-mounted permanent magnet motor rotor's hot pressing equipment.

\section{Rotor Strength Design Considering Assembly Pressing Force}

\subsection{Analysis of the Influence of Assembly Pressing Force on the Rotor Strength}

In order to facilitate researching the influence of assembly pressing force on the rotor strength of the cylindrical magnet surface-mounted permanent magnet motor, combined with the geometrical shape of the motor rotor, constraints and external forces, the forces on magnet, sleeve and short shaft are analyzed by using the basic theory of the elastic mechanics space axisymmetric problem, as follows [2]:

Based on the generalized Hooke's law, the expressions of stresses and strain are obtained as follows when only the assembly pressing force is considered:

$$
\left\{\begin{array}{l}
\sigma_{r}=\frac{E\left(\varepsilon_{r}+\mu \varepsilon_{\theta}\right)+\mu(1+\mu) P}{1-\mu^{2}} \\
\sigma_{\theta}=\frac{E\left(\varepsilon_{\theta}+\mu \varepsilon_{r}\right)+\mu(1+\mu) P}{1-\mu^{2}}
\end{array}\right.
$$

where $\sigma_{r}$ and $\sigma_{\theta}$ are radial and tangential stress; $\varepsilon_{r}$ and $\varepsilon_{\theta}$ are radial and tangential linear strains, $\varepsilon_{r}=\frac{\mathrm{d} u}{\mathrm{~d} r}, \varepsilon_{\theta}=\frac{u}{r}, u$ is radial displacement; $E$ is elastic modulus; $\mu$ is Poisson's ratio; $P$ is pressure on the end face of short shaft, which is from assembly pressing force and related to the area of the end face.

Then, the second-order differential equation of displacement is obtained from the equilibrium differential equation as follows:

$$
\frac{\mathrm{d}^{2} u}{\mathrm{~d} r^{2}}+\frac{1}{r} \frac{\mathrm{d} u}{\mathrm{~d} r}-\frac{u}{r^{2}}=0
$$

Therefore, the displacement $u$ can be expressed as:

$$
u=M_{i} r+\frac{N_{i}}{r}
$$

where, $M_{i}$ and $N_{i}$ are undetermined coefficients, when $i=h, u$ is the sleeve displacement; when $i=m, u$ is the magnet displacement; when $i=z, u$ is the short shaft displacement.

Correspondingly, the expressions of radial stress and tangential stress are:

$$
\left\{\begin{array}{l}
\sigma_{r}=\frac{E\left((1+\mu) M_{i}-(1-\mu) \frac{N_{i}}{r^{2}}\right)+\mu(1+\mu) P}{1-\mu^{2}} \\
\sigma_{\theta}=\frac{E\left((1+\mu) M_{i}+(1-\mu) \frac{N_{i}}{r^{2}}\right)+\mu(1+\mu) P}{1-\mu^{2}}
\end{array}\right.
$$

Because of $P=\mu\left(\sigma_{\theta}+\sigma_{r}\right)$, so the undermined coefficient $M_{i}$ can be obtained:

$$
M_{i}=\frac{(1-2 \mu)(1+\mu) P}{2 E \mu}
$$


The undetermined coefficient $N_{i}$ is determined by the boundary conditions of the rotor's various parts, and the coefficient will be different for different parts. The specific analysis results are as follows:

- Sleeve: Because there is no force on the outer wall $R_{h o}$ of the sleeve, the coefficient $N_{h}$ in the sleeve stresses equation can be obtained:

$$
N_{h}=\frac{P\left(1+\mu_{h}\right) R_{h o}^{2}}{2 E_{h} \mu_{h}}
$$

- Magnet: Because the magnet is solid, there is no displacement at the inner diameter of the magnet, that is $\left.u_{m}{ }^{*}\right|_{r=0}=0$, so the coefficient $N_{m}$ in the magnet stresses equation can be obtained:

$$
N_{m}=0
$$

- Short shaft: The boundary conditions of the short shaft are the same as the magnet, so the undetermined coefficient $N_{z}$ is also 0 .

From the above analysis, it can be seen that when considering the assembly pressing force, the displacement expression and stresses expression of the sleeve are:

$$
\begin{aligned}
u_{h}{ }^{*}= & \frac{P\left(1+\mu_{h}\right)}{2 E_{h} \mu_{h}}\left(\left(1-2 \mu_{h}\right) r+\frac{R_{h o}{ }^{2}}{r}\right) \\
& \left\{\begin{array}{l}
\sigma_{r h}{ }^{*}=\frac{P}{2 \mu_{h}}\left(1-\frac{R_{h o}{ }^{2}}{r^{2}}\right) \\
\sigma_{\theta h}{ }^{*}=\frac{P}{2 \mu_{h}}\left(1+\frac{R_{h o}{ }^{2}}{r^{2}}\right)
\end{array}\right.
\end{aligned}
$$

The displacement expression and stresses expression of the short shaft and the magnet are:

$$
\begin{gathered}
u_{i}{ }^{*}=\frac{\left(1-2 \mu_{i}\right)\left(1+\mu_{i}\right) P}{2 E_{i} \mu_{i}} r \quad i=m, z \\
{\sigma_{r i}}^{*}=\sigma_{\theta i}{ }^{*}=\frac{P}{2 \mu_{i}} \quad i=m, z
\end{gathered}
$$

The variation $\Delta \delta$ of interference $\delta$ between magnet and sleeve is:

$$
\begin{aligned}
\Delta \delta & =\left.u_{h}{ }^{*}\right|_{r=R_{h i}}-\left.u_{m}{ }^{*}\right|_{r=R_{m o}} \\
& =\frac{P\left(1+\mu_{h}\right)}{2 E_{h} \mu_{h}}\left(\left(1-2 \mu_{h}\right) R_{h i}+\frac{R_{h o}{ }^{2}}{R_{h i}}\right)-\frac{P\left(1+\mu_{m}\right)\left(1-2 \mu_{m}\right)}{2 E_{m} \mu_{m}} R_{m o}
\end{aligned}
$$

The variation $\Delta \delta_{0}$ of interference $\delta_{0}$ between short shaft and sleeve is:

$$
\begin{aligned}
\Delta \delta_{0} & =\left.u_{h}{ }^{*}\right|_{r=R_{h i}}-\left.u_{z}{ }^{*}\right|_{r=R_{z o}} \\
& =\frac{P\left(1+\mu_{h}\right)}{2 E_{h} \mu_{h}}\left(\left(1-2 \mu_{h}\right) R_{h i}+\frac{R_{h o}{ }^{2}}{R_{h i}}\right)-\frac{P\left(1+\mu_{z}\right)\left(1-2 \mu_{z}\right)}{2 E_{z} \mu_{z}} R_{z o}
\end{aligned}
$$

Therefore, when considering the assembly pressing force, the interference between magnet and sleeve is $\delta+\Delta \delta$, the interference between short shaft and sleeve is $\delta_{0}+\Delta \delta_{0}$, and the Mises stress of each part will change from Equation (14) to (15):

$$
\begin{aligned}
& \sigma=\sqrt{\frac{1}{2}\left[\left(\sigma_{r i}-\sigma_{\theta i}\right)^{2}+\left(\sigma_{\theta i}-\sigma_{z i}\right)^{2}+\left(\sigma_{z i}-\sigma_{r i}\right)^{2}\right]} \\
& \sigma=\sqrt{\frac{1}{2}\left[\left(\left(\sigma_{r i}+\sigma_{r i}^{*}\right)-\left(\sigma_{\theta i}+\sigma_{\theta i}{ }^{*}\right)\right)^{2}+\left(\left(\sigma_{\theta i}+\sigma_{\theta i}{ }^{*}\right)-\left(\sigma_{z i}+P\right)\right)^{2}+\left(\left(\sigma_{z i}+P\right)-\left(\sigma_{r i}+\sigma_{r i}{ }^{*}\right)\right)^{2}\right]}
\end{aligned}
$$

Based on the above analysis, it can be seen that the assembly pressing force will affect interference and stresses of the rotor's various parts, and the greater the assembly pressing force, the more serious the influence. Besides, the influence is related to the structural size 
and material properties of the rotor. Therefore, in order to ensure design's accuracy and reliability of the rotor strength, the assembly pressing force must be included in advance.

\subsection{Design Method}

The assembly pressing force causes the mutual influence of the rotor's each part to be more significant in the axial direction. Therefore, the rotor strength's design considering the influence of assembly pressing force needs to be modeled on the whole shaft. When designing, comprehensively considering the influence of assembly pressing force, centrifugal force, temperature and interference, check the stresses of the rotor's various part in high-speed cold state and high-speed hot state, and determine the optimal interference range between magnet and sleeve, as well as between short shaft and sleeve.

On the basis of the above analysis, this article proposes a rotor strength's design method with high structural stability considering the influence of assembly pressing force. The design flow chart is shown in Figure 3. The specific steps are as follows:

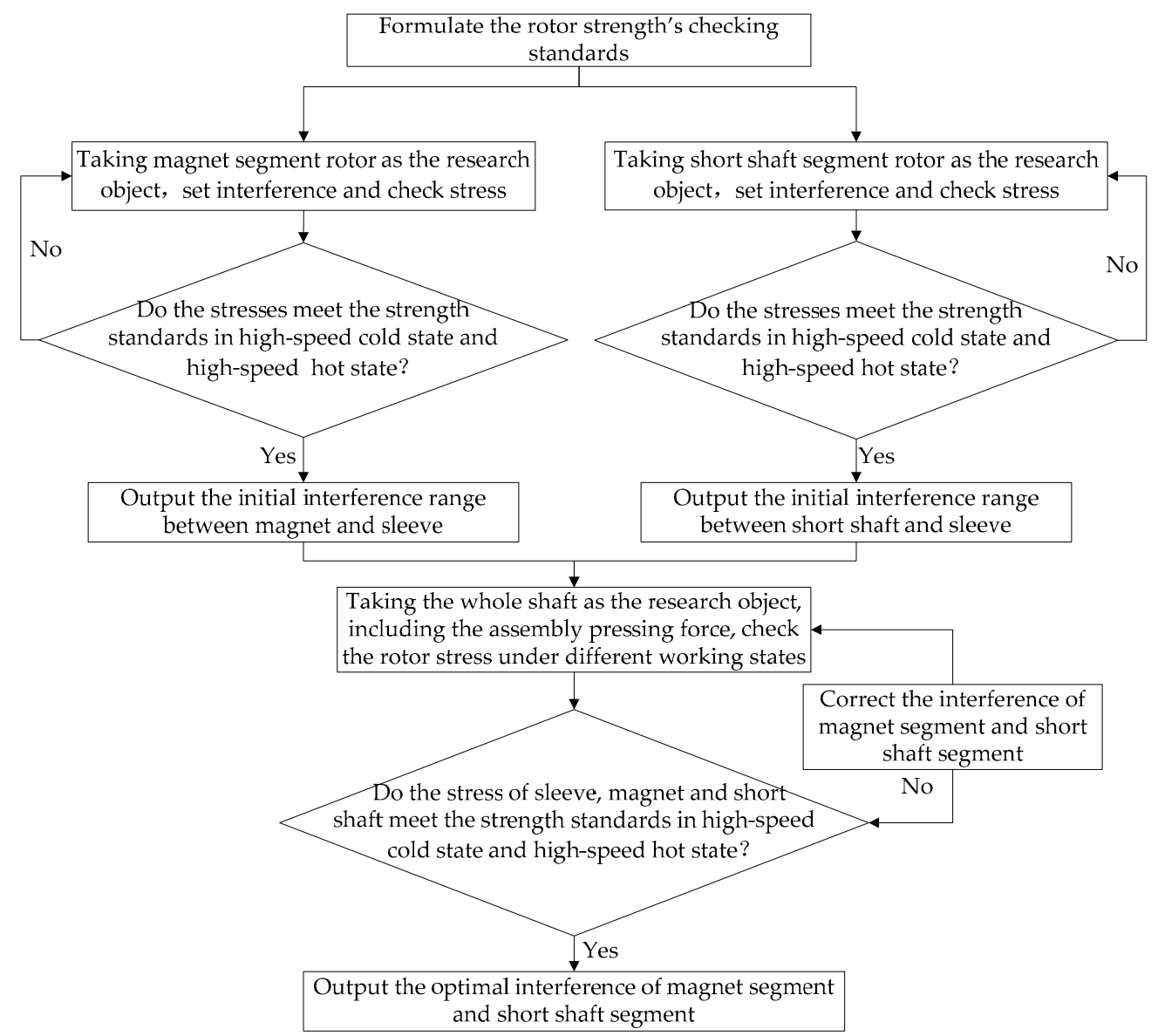

Figure 3. The rotor strength's design method considering the influence of assembly pressing force.

(1) According to the safe and reliable conditions of the rotor structure, formulate the rotor strength's checking standards.

- The components do not exceed the strength limit. For the plastic material, such as short shaft and magnet, the strength limit is the material yield strength. But for the brittle material, the strength limit is the material tensile strength.

- For the components assembled together, there must be no loosening in the mating surface, which means that the internal components must be always pressed, and the normal stress always is less than 0 on the outer surface of the internal components. 
(2) Taking the magnet segment rotor and the short shaft segment rotor as the research objects respectively, based on the strength's checking standards, design the initial interference range of each segment. It should be noted that this step is only to obtain an initial interference range, and is not limited to using the method in the flow chart of Figure 3, but this method will reduce the iterative process of the optimal interference design. Specifically, ensure that all of components meet the strength standards, and obtain the maximum and minimum value of the interference by continuously checking each component's stresses.

(3) Taking the whole shaft as the research object, including the assembly pressing force, check whether the rotor stresses under different working states and different initial interference matching states satisfy the strength standards. If so, the initial interference is the optimal interference; if not, correct each segment's interference based on the whole shaft model.

\section{Case Analysis}

\subsection{Finite Element Design}

Taking a $100 \mathrm{~kW} / 30,000 \mathrm{rpm}$ high-speed permanent magnet motor as an example, based on the above-mentioned rotor strength design method, by finite element method, considering the assembly pressing force of $30 \mathrm{MPa}$, check the rotor stresses of cold state $25^{\circ} \mathrm{C}$ and hot state $85^{\circ} \mathrm{C}$ at 1.2 times of the rated speed, and design the optimal interference range between magnet and sleeve, as well as between short shaft and sleeve. The rotor's finite element model is shown in Figure 4, and the rotor's outer diameters are $83 \mathrm{~mm}$, and the sleeve's thickness is $4.5 \mathrm{~mm}$. The properties of various parts are shown in Table 1.

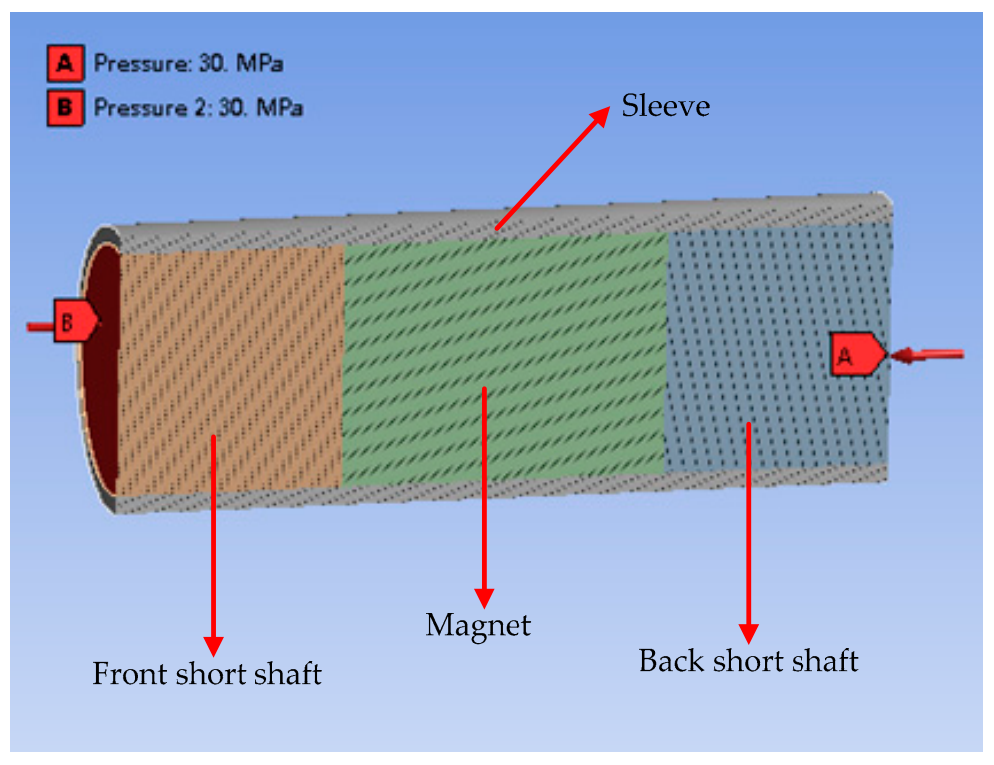

Figure 4. The finite element model of 30,000 rpm high-speed permanent magnet motor rotor.

Table 1. Materials and properties of various rotor parts.

\begin{tabular}{ccccc}
\hline & $\begin{array}{c}\text { Density } \\
\left(\mathbf{k g} / \mathbf{m}^{\mathbf{3}}\right)\end{array}$ & $\begin{array}{c}\text { Elastic } \\
\text { Modulus (GPa) }\end{array}$ & Poisson's Ratio & $\begin{array}{c}\text { Thermal } \\
\text { Expansion } \\
\left.\text { Coefficient (1/ }{ }^{\circ} \mathbf{C}\right)\end{array}$ \\
\hline $\begin{array}{c}\text { Sleeve } \\
\text { Magnet }\end{array}$ & 8240 & 199 & 0.24 & $1.325 \times 10^{-5}$ \\
$\begin{array}{c}\text { Front and back } \\
\text { short shaft }\end{array}$ & 7600 & 160 & 0.24 & $9 \times 10^{-6}$ \\
\hline
\end{tabular}


Considering the strength limit of the rotor's various parts provided by the manufacturer and the requirements for not loosening during operation, the strength's checking standards are formulated as follows:

(1) The Mises stress of the sleeve is less than the material yield strength of $950 \mathrm{MPa}$.

(2) The radial stress and tangential stress of the magnet are both less than the material tensile strength of $60 \mathrm{MPa}$, and it is necessary to ensure that its outer surface is always under pressure, that is, the normal stress on the outer surface is negative.

(3) The Mises stress of the short shaft is less than the material yield strength of $400 \mathrm{MPa}$, and it is necessary to ensure that the outer surface is always under pressure, that is, the normal stress on the outer surface is negative.

Based on the above strength standards, respectively check the rotor stresses of magnet segment and short shaft segment under high-speed cold state $\left(25^{\circ} \mathrm{C}, 36,000 \mathrm{rpm}\right)$ and high-speed hot state $\left(85^{\circ} \mathrm{C}, 36,000 \mathrm{rpm}\right)$ to obtain that the range of initial interference $\delta$ between magnet and sleeve is $0.208-0.343 \mathrm{~mm}$, and the range of initial interference $\delta 0$ between short shaft and sleeve is $0.059-0.327 \mathrm{~mm}$.

Taking the whole shaft in Figure 4 as the research object, considering the assembly pressing force as the boundary condition (see pressure A and pressure B of Figure 4), substituting the initial interference of magnet segment and short shaft segment, the rotor stresses under high-speed cold state and high-speed hot state with different interference matching states are checked. The specific results are shown in Table 2, and the stresses comparative analysis of various parts under different interference matching schemes are shown in Figures 5-7.

Table 2. Simulation results of the whole shaft strength under the initial interference.

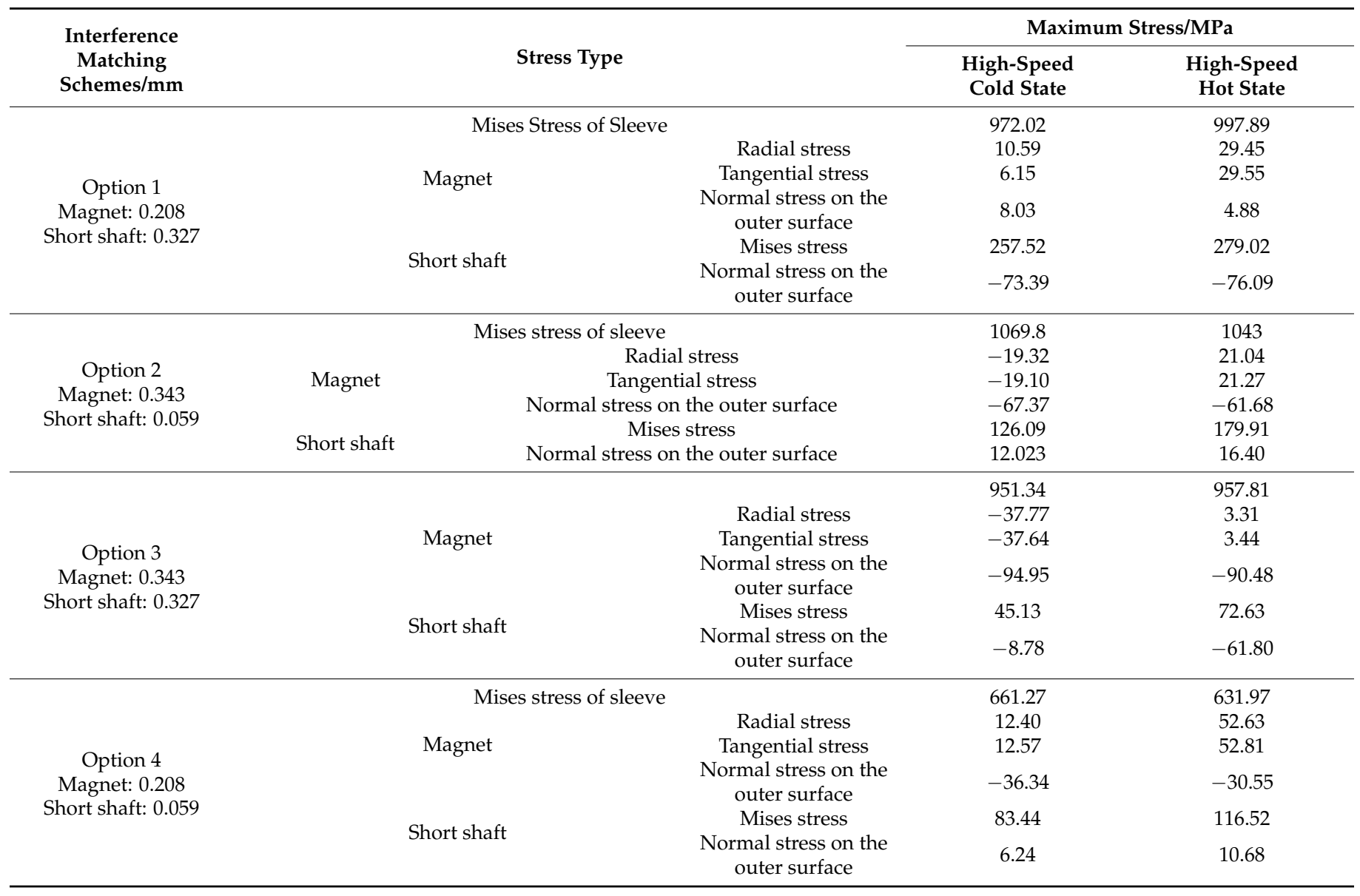




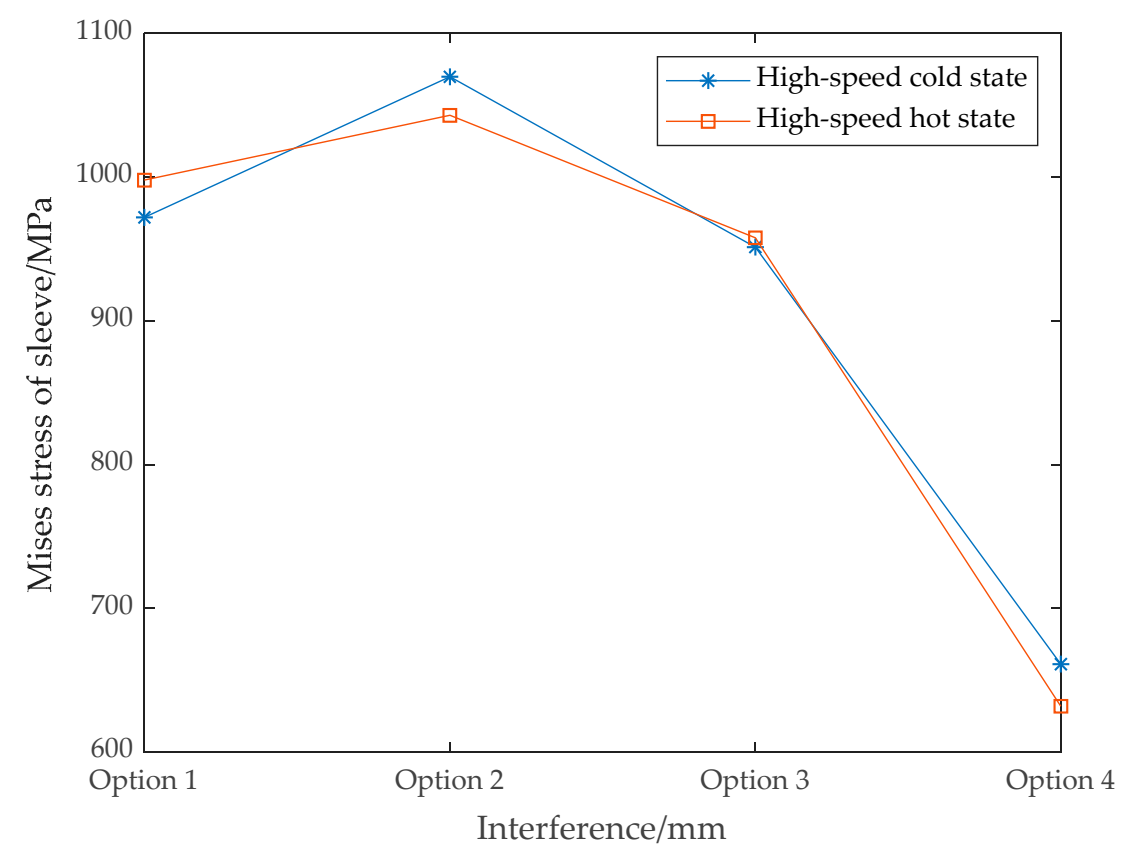

Figure 5. Mises stresses of sleeve under the initial interference.

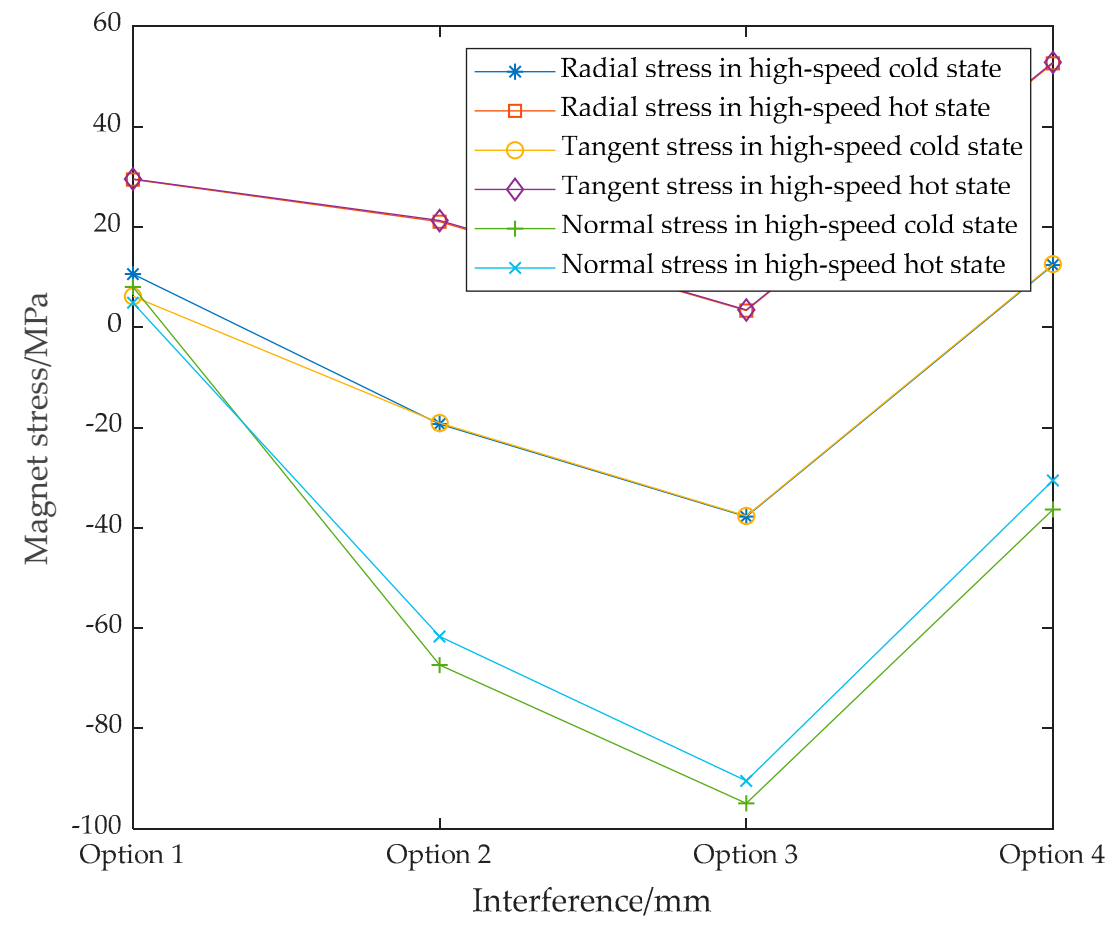

Figure 6. Magnet stresses under the initial interference.

Analyzing the data in Table 2 and comparing the results of Figures 5-7, we can see that:

(1) When magnet segment has the minimum interference $\delta(0.208 \mathrm{~mm})$ and short shaft segment has the maximum interference $\delta 0(0.327 \mathrm{~mm})$, the stresses of magnet and short shaft under high-speed cold state and high-speed hot state meet the strength requirements and the outer surface of short shaft is fully compressed, but the sleeve stress has exceeded $950 \mathrm{MPa}$, and the outer surface of magnet has not been fully compressed.

(2) When magnet segment has the maximum interference $\delta(0.343 \mathrm{~mm})$ and short shaft segment has the minimum interference $\delta 0(0.059 \mathrm{~mm})$, the stresses of magnet and short shaft under high-speed cold state and high-speed hot state meet the strength 
requirements and the outer surface of magnet is fully compressed, but the sleeve stress has exceeded $950 \mathrm{MPa}$, and the outer surface of short shaft is not fully compressed.

(3) When magnet segment has the maximum interference $\delta(0.343 \mathrm{~mm})$ and short shaft segment has the maximum interference $\delta 0(0.327 \mathrm{~mm})$, the outer surface of magnet and short shaft are fully compressed and the stresses under high-speed cold state and high-speed hot state meet the strength requirements, but the sleeve stress has exceeded $950 \mathrm{MPa}$.

(4) When magnet segment has the minimum interference $\delta(0.208 \mathrm{~mm})$ and short shaft segment has the minimum interference $\delta 0(0.059 \mathrm{~mm})$, the stresses of sleeve, magnet and short shaft under high-speed cold state and high-speed hot state meet the strength requirements and the outer surface of magnet is fully compressed, but the outer surface of short shaft is not fully compressed.

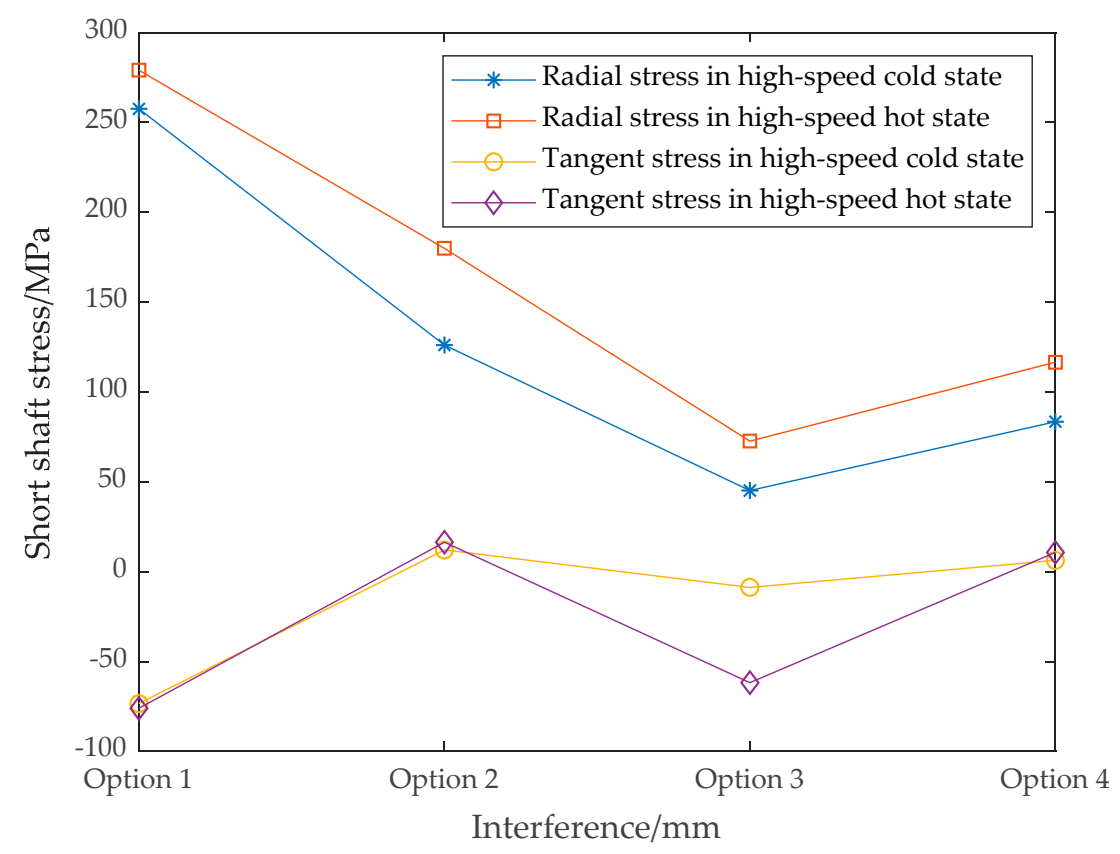

Figure 7. Short shaft stresses under the initial interference.

It can be seen from the analysis results that the initial interference range based on magnet segment and short shaft segment is not suitable for the whole shaft model when the assembly pressing force is considered. Therefore, it is necessary to optimize the interference of each segment based on the whole shaft model, which ensures that all parts of the rotor can meet the strength standards under different working states and different initial interference matching states. Finally, obtain that the optimal interference $\delta$ between magnet and sleeve is $0.283-0.332 \mathrm{~mm}$, and the optimal interference $\delta 0$ between short shaft and sleeve is $0.287-0.316 \mathrm{~mm}$. The stresses of various part under different working conditions and optimal interference are shown in Table 3 and the stresses comparative analysis of various part under different interference matching schemes is shown in Figures 8-10, and the stresses distribution diagrams of various part under dangerous state are shown in Figures 11-15. 
Table 3. Simulation results of the whole shaft strength under the optimal interference.

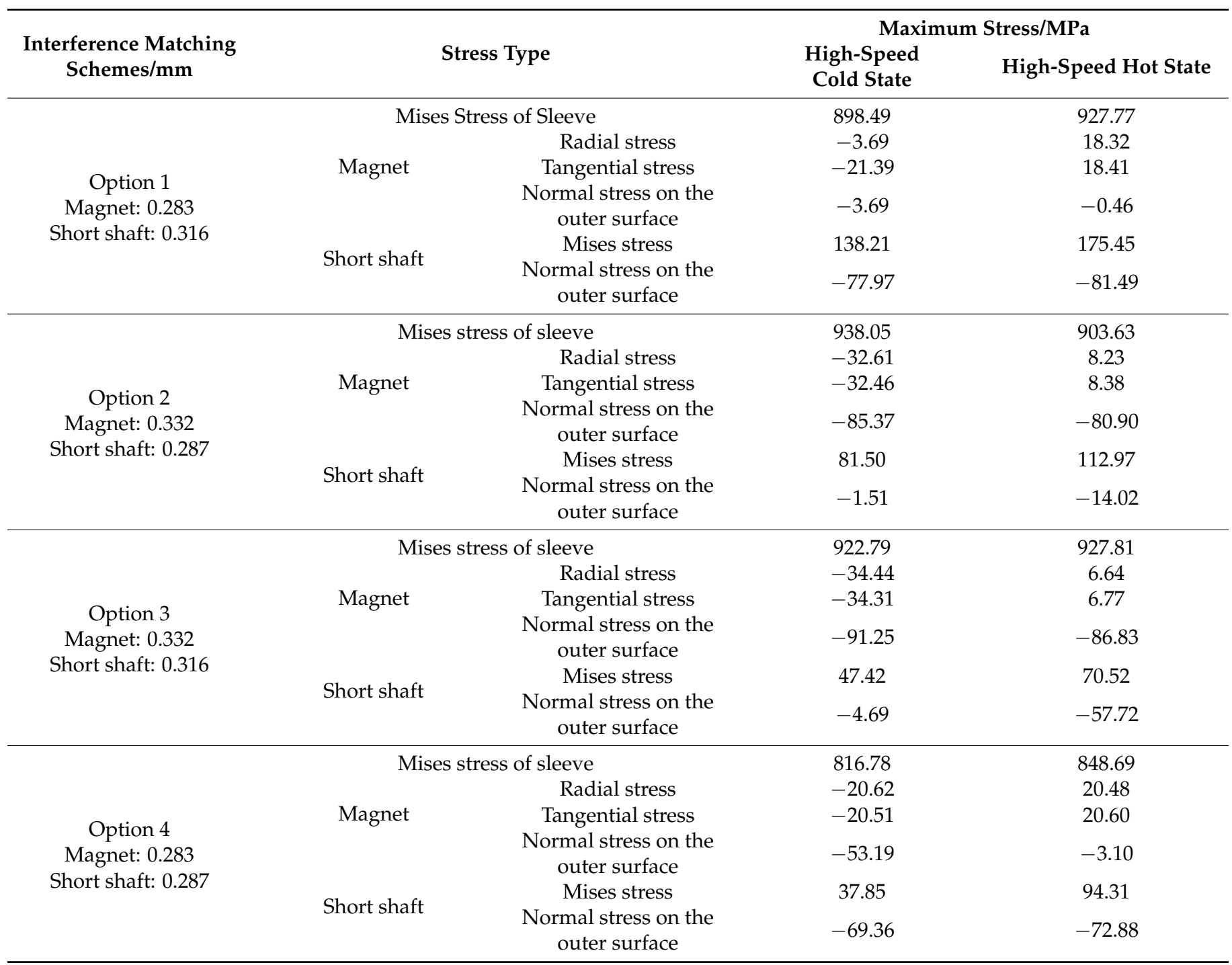

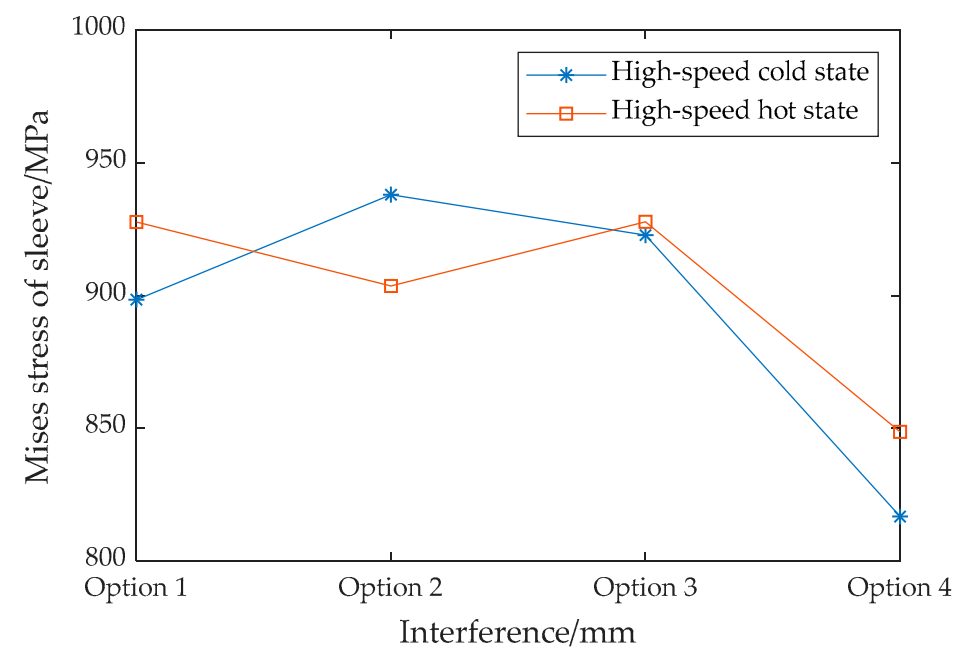

Figure 8. Mises stresses of sleeve under the optimal interference. 


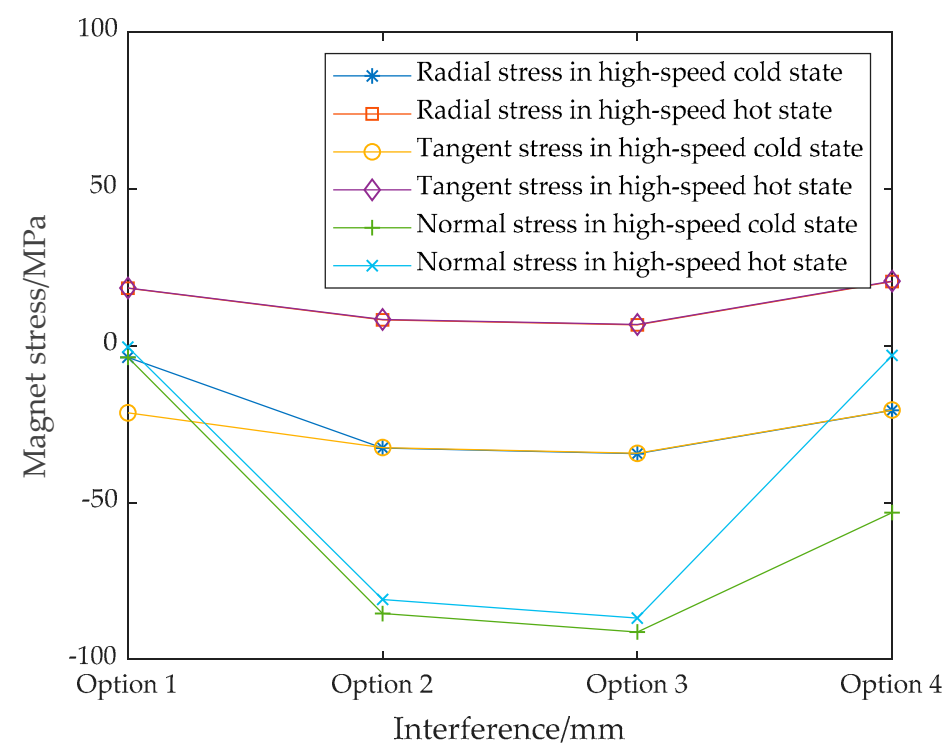

Figure 9. Magnet stresses under the optimal interference.

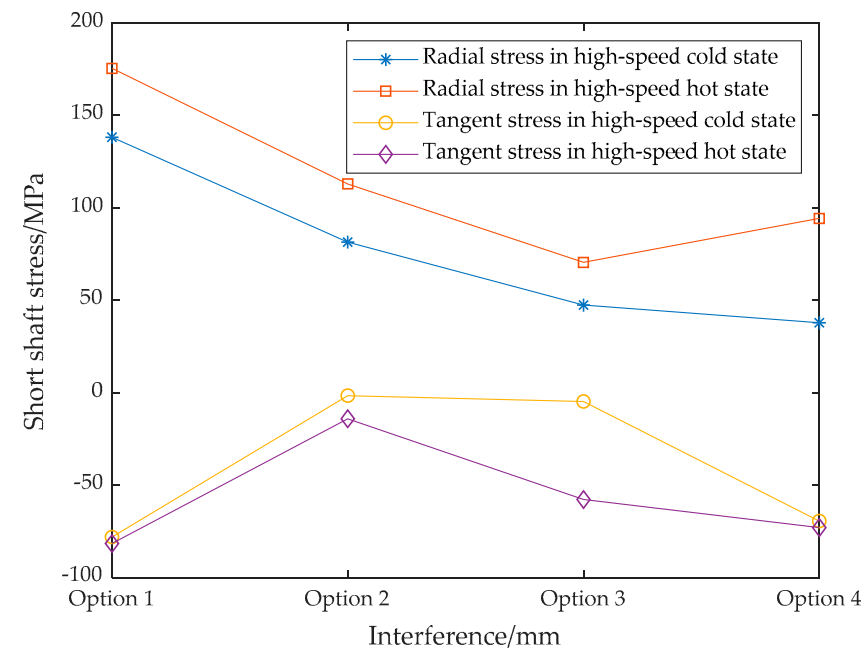

Figure 10. Short shaft stresses under the optimal interference.
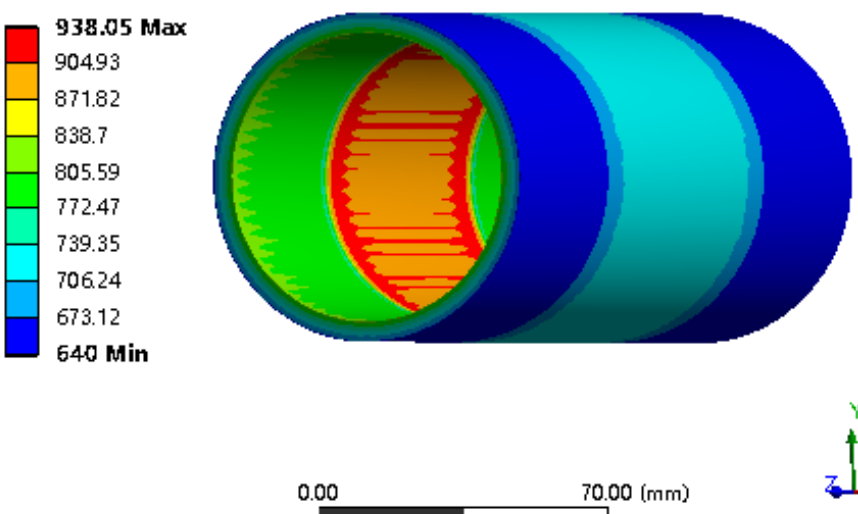

$70.00(\mathrm{~mm})$

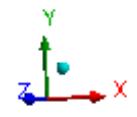

35.00

Figure 11. The most dangerous Mises stress distribution of sleeve. 


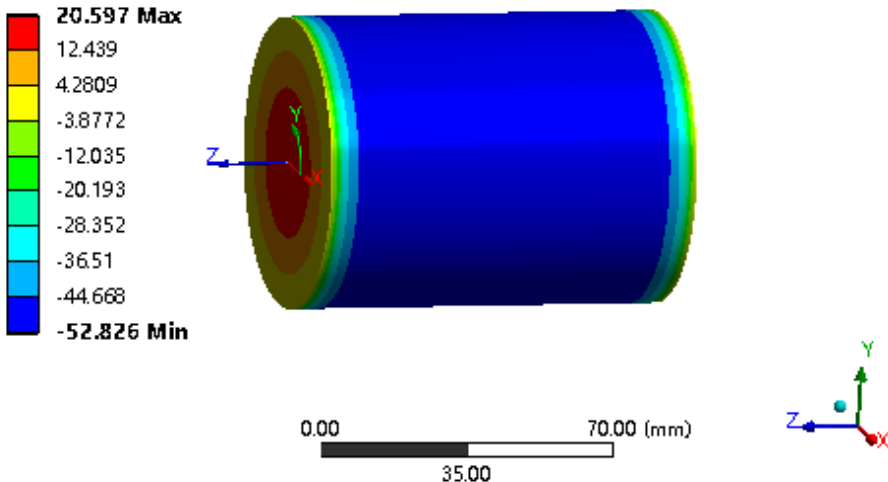

Figure 12. The most dangerous stress distribution of magnet.

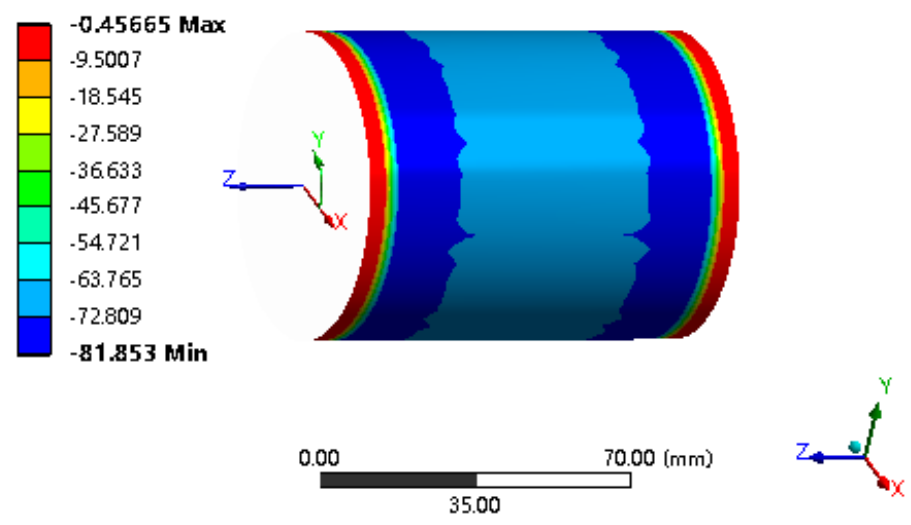

Figure 13. The normal stress distribution of magnet under the most easily loosening state.
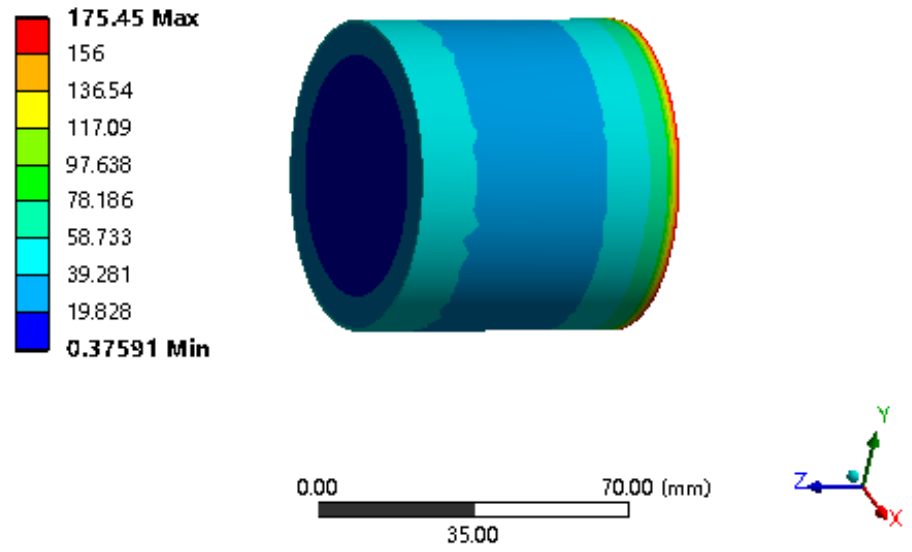

Figure 14. The most dangerous stress distribution of short shaft. 

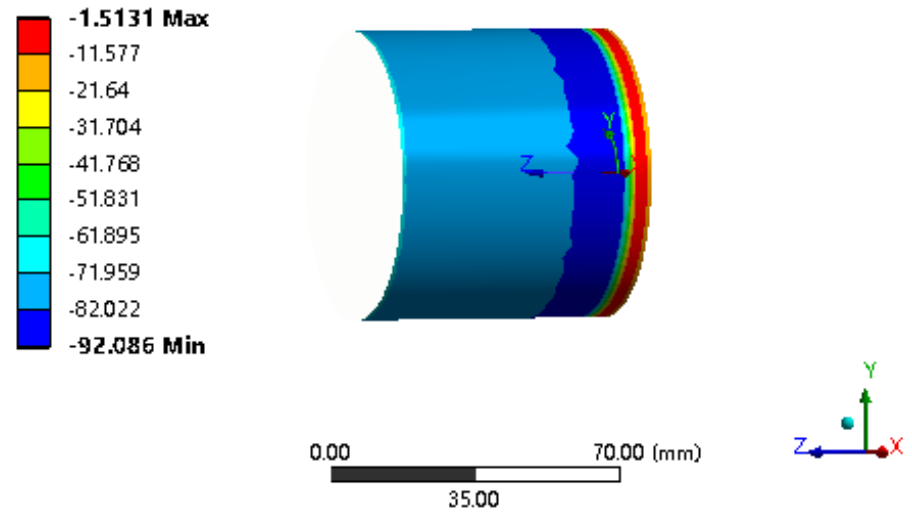

Figure 15. The normal stress distribution of short shaft under the most easily loosening state.

It can be seen from the stresses data in Table 3, and the stresses comparative analysis in Figures 8-10, and the stresses distribution diagrams in Figures 11-15 that:

(1) The most dangerous stress of sleeve is $938.05 \mathrm{MPa}$, which occurs on the inner surface of sleeve when magnet segment has the maximum interference $\delta(0.332 \mathrm{~mm})$ and short shaft segment has the minimum interference $\delta 0(0.287 \mathrm{~mm})$ under high-speed cold state, and the margin to yield limit is $1.26 \%$.

(2) The maximum stress of magnet is $20.6 \mathrm{MPa}$, which occurs on the core of magnet when magnet segment has the minimum interference $\delta(0.283 \mathrm{~mm})$ and short shaft segment has the minimum interference $\delta 0(0.287 \mathrm{~mm})$ under high-speed hot state, and the margin to tensile limit is $65.67 \%$, with sufficient safety margin.

(3) The most easily loosening point of magnet occurs on the contact surface between magnet and front or back short shaft when magnet segment has the minimum interference $\delta(0.283 \mathrm{~mm})$ and short shaft segment has the maximum interference $\delta 0(0.316 \mathrm{~mm})$ under high-speed hot state, and the normal stress on the outer surface is $-0.46 \mathrm{MPa}$.

(4) The maximum stress of short shaft is $175.45 \mathrm{MPa}$, which occurs on the contact surface's brink between front or back short shaft and magnet when magnet segment has the minimum interference $\delta(0.283 \mathrm{~mm})$ and short shaft segment has the maximum interference $\delta 0(0.316 \mathrm{~mm})$ under high-speed hot state, and the margin to tensile limit is $56.14 \%$, with sufficient safety margin.

(5) The most easily loosening point of short shaft occurs on the contact surface between front or back short shaft and magnet when magnet segment has the maximum interference $\delta(0.332 \mathrm{~mm})$ and short shaft segment has the minimum interference $\delta 0$ $(0.287 \mathrm{~mm})$ under high-speed cold state, and the normal stress on the outer surface is $-1.51 \mathrm{MPa}$.

\subsection{Over-Speed Experiment}

In order to verify the rotor strength, an over-speed rotor is made for over-speed experiment. The physical diagram and cross section of the over-speed rotor are shown in Figure 16.

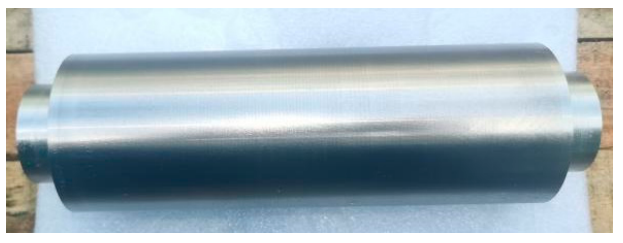

(a)

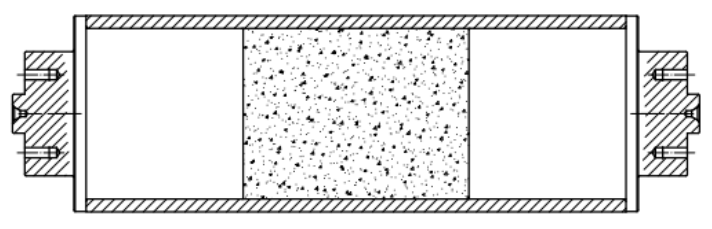

(b)

Figure 16. Over-speed rotor: (a) physical rotor; (b) cross-sectional view. 
Before the over-speed experiment, the imbalance and different axial positions' outer diameter of the over-speed rotor are measured and recorded to assess whether the rotor strength is qualified.

During the over-speed experiment, the one side of the over-speed rotor is fastened to high-speed shaft of the test bench with four bolts, then the rotor gradually speeds up to $36,000 \mathrm{rpm}$ and runs stably for $2 \mathrm{~min}$ to complete the over-speed experiment. The experimental site is shown in Figure 17, and the speed curve of the rotor during the experiment are shown in Figure 18.

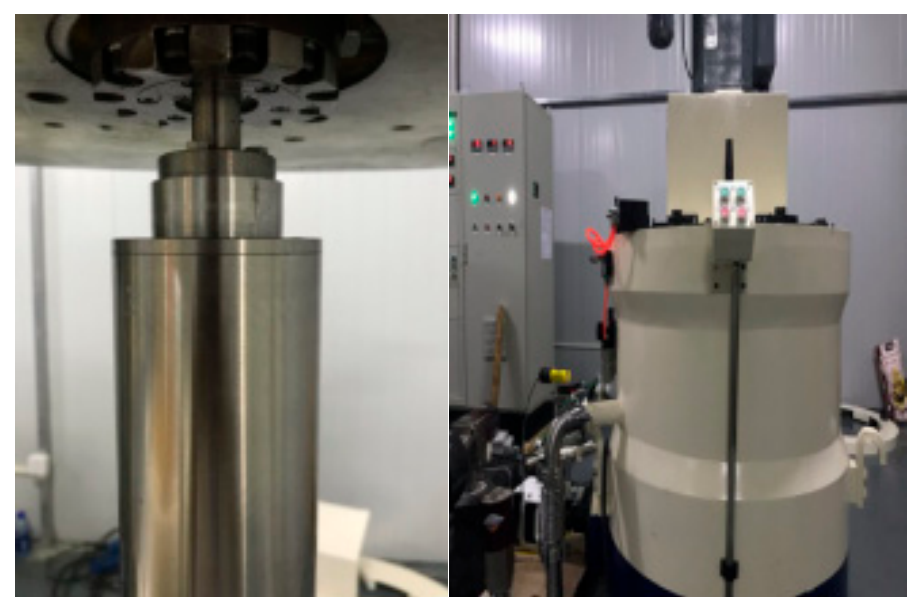

Figure 17. Over-speed experiment's site.

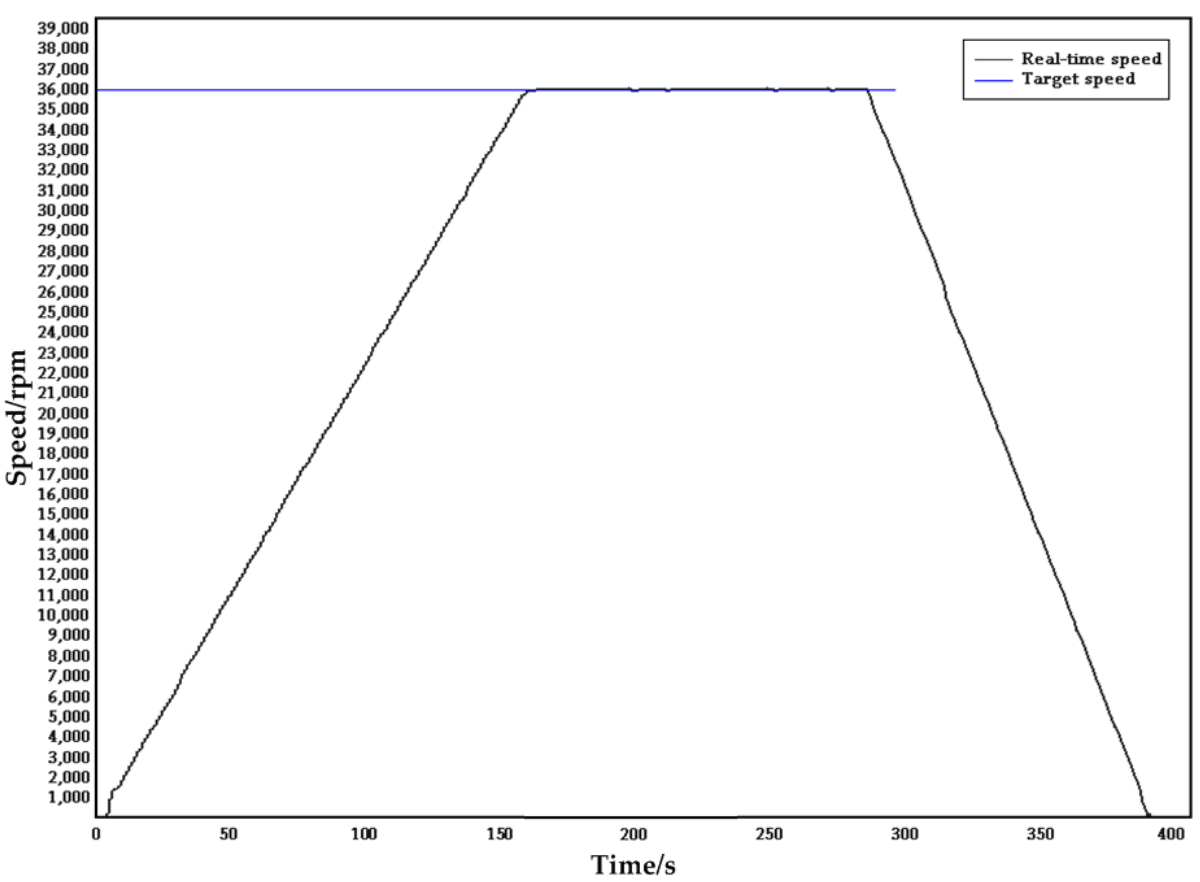

Figure 18. The speed and vibration curve during the over-speed experiment.

As can be seen from Figure 18, the over-speed rotor can stably speed up to the rated speed's 1.2 times (36,000 rpm) and maintain two minutes and have no abnormalities during the deceleration process. Simultaneously, compared the data after the experiment with before the experiment, the maximum change in the outer diameter of the rotor is $0.01 \mathrm{~mm}$ and the rotor's imbalance almost has not changed, which indicates that the rotor strength can meet the requirements of max-speed 30,000 rpm, and the structural stability is high. 


\section{Conclusions}

Based on the rotor strength's requirements in the full range of working range, combined with the engineering practice, comprehensively considering the assembly pressing force, speed, temperature and interference, this paper proposes a rotor strength's design method with high structural stability, which improves the accuracy of rotor interference's design and the reliability of shaft's structural strength. Based on this method, the $100 \mathrm{~kW} / 30,000 \mathrm{rpm}$ rotor is designed, and obtain that the range of interference $\delta$ between magnet and sleeve is $0.283-0.332 \mathrm{~mm}$, and the range of interference $\delta 0$ between short shaft and sleeve is $0.287-0.316 \mathrm{~mm}$, which can ensure that all of stresses on the magnet, short shaft and sleeve meet the strength's checking standards. Finally, the over-speed experiment is carried out, and the rotor size and imbalance do not change significantly before and after the experiment, which meets the requirements of engineering.

At the same time, based on the strength's research of the cylindrical magnet surfacemounted high-speed permanent magnet synchronous motor rotor with taking the whole shaft as the research object in this paper, the following conclusions can be obtained, which plays a guiding role for the engineering design.

(1) The most dangerous situation of sleeve is that magnet segment has the maximum interference and short shaft segment has the minimum interference under high-speed cold state, and the dangerous segment occurs on magnet.

(2) High-speed hot state is more dangerous than high-speed cold state for magnet's stresses. The most dangerous stress of magnet is that all of magnet segment and short shaft segment have the minimum interference under high-speed hot state, and the more dangerous stress is that magnet segment has the minimum interference and short shaft segment has the maximum interference.

(3) Short shaft's stresses under high-speed hot state are also more dangerous than under high-speed cold state. The most dangerous stress is that magnet segment has the minimum interference and short shaft segment has the maximum interference under high-speed hot state.

(4) Both magnet and short shaft are easy to loosen at the interface between them. Magnet is easy to loosen under hot state, and short shaft is easy to loosen under cold state. The most easily loosening point of magnet is that magnet has the minimum interference and short shaft has the maximum interference under high-speed hot state. The most easily loosening point of short shaft is that short shaft has the minimum interference and magnet has the maximum interference under high-speed cold state.

Author Contributions: Conceptualization, Y.H. and L.L.; methodology, Y.H.; software, Y.H.; validation, Y.H., W.G. and S.W.; formal analysis, Y.H.; investigation, Y.H.; resources, Y.H.; data curation, Y.H.; writing—original draft preparation, Y.H.; writing—review and editing, Y.H.; visualization, Y.H.; supervision, L.L.; project administration, L.L.; funding acquisition, L.L. All authors have read and agreed to the published version of the manuscript.

Funding: This research received no external funding.

Institutional Review Board Statement: Not applicable.

Informed Consent Statement: Not applicable.

Data Availability Statement: Not applicable, all research data and results have been given in the paper.

Conflicts of Interest: The authors declare no conflict of interest. 


\section{References}

1. Chen, L.L.; Zhu, C.S.; Zhong, Z.; Liu, B.; Wan, A. Rotor strength analysis for high-speed segmented surface-mounted permanent magnet synchronous machines. IET Electr. Power Appl. 2018, 12, 979-990. [CrossRef]

2. Chen, L.; Zhu, C. Rotor Strength Analysis for High Speed Permanent Magnet Machines. In Proceedings of the 2014 17th International Conference on Electrical Machines and Systems (ICEMS), Hangzhou, China, 22-25 October 2014; pp. 65-69.

3. Xiao, J.C.; Xiao, S.H.; Wu, H. Design and strength analysis of ultra-high speed permanent magnet DC rotor. Adv. Mater. Res. 2011, 188, 481-486. [CrossRef]

4. Du, G.; Huang, N. Multiphysics analysis of high-speed permanent magnet generators for waste heat application. IET Electr. Syst. Transport. 2017, 7, 278-286. [CrossRef]

5. Hong, D.K.; Woo, B.C.; Jeong, Y.H.; Koo, D.H.; Ahn, C.W. Development of an ultra high speed permanent magnet synchronous motor. Int. J. Precis. Eng. Manuf. 2013, 14, 493-499. [CrossRef]

6. Borisavljevic, A.; Polinder, H.; Ferreira, J.A. Enclosure design for a high-speed permanent magnet rotor. In Proceedings of the IET International Conference on Power Electronics, Machines and Drives, Brighton, UK, 21-24 June 2010; pp. 1-6.

7. Zwyssig, C.; Kolar, J.W. Design of a 100W, 500000rpm Permanent-Magnet Generator for Mesoscale Gas Turbines. In Proceedings of the IEEE-IAS, Hong Kong, China, 2-6 October 2005; pp. 253-260.

8. Zhou, Y.; Fang, J. Strength Analysis of Enclosure for a High-Speed Permanent Magnet Rotor. AASRI Procedia 2012, 3, 652-660. [CrossRef]

9. Huang, Z.; Fang, J. Multi-Physics Design and Optimization of High Speed Permanent Magnet Electrical Machines for Air Blower Applications. IEEE Trans. Ind. Electron. 2016, 63, 2766-2774. [CrossRef]

10. Fang, H.; Qu, R.; Li, J.; Zheng, P.; Fan, X. Rotor Design for High Speed High-Power Permanent-Magnet Synchronous Machines. IEEE Trans. Ind. Appl. 2017, 53, 3411-3419. [CrossRef] 